\title{
Transanal Total Mesorectal Excision for Low Rectal Cancer
}

\author{
Chang Woo Kim, M.D. ${ }^{1}$, Yoona Chung, M.D. ${ }^{1}$, Sun Jin Park, M.D., Ph.D. ${ }^{2}$ Kil Yeon Lee, M.D., Ph.D. ${ }^{2}$, Suk-Hwan Lee, M.D., Ph.D. ${ }^{1}$ \\ Department of Surgery, 'Kyung Hee University Hospital at Gangdong, Kyung Hee University School of Medicine, ${ }^{2}$ Kyung Hee Medical Center, Kyung Hee \\ University School of Medicine, Seoul, Korea
}

\begin{abstract}
A 70-year-old female patient was diagnosed with low rectal adenocarcinoma (cT3N2) based on the initial CT and MRI. The patient underwent neoadjuvant chemoradiotherapy consisting of short course radiotherapy with 5-fluorouracil (5-FU) and leucovorin (LV) chemotherapy. Three additional cycles of simplified infusional 5-FU/LV were given every 2 weeks to the patient during the resting period (8 weeks) before surgery. For transanal TME, a purse-string suture of the distal rectum was performed just above the dentate line. Transanal circumferential dissection including the mesorectum was performed from the dentate line until the peritoneal reflection. Thereafter, laparoscopic dissection was conducted using the medial to lateral approach and the inferior mesenteric artery was ligated at the pedicle. Lateral detachment and splenic flexure mobilization were completed. After full mobilization of the distal transverse colon and rectum, the specimen was retrieved through the anus and resected. Colo-anal anastomosis was performed by the hand-sewn method. A closed suction drain was inserted into the pelvis. We also demonstrate our procedure for transanal TME using a short video clip.
\end{abstract}

Received June 1, 2016

Accepted June 9, 2016

Corresponding author

Suk-Hwan Lee

Department of Surgery, Kyung Hee

University Hospital at Gangdong,

Kyung Hee University School

of Medicine, 892 Dongnam-ro,

Gangdong-gu, Seoul 05278, Korea

Tel: +82-2-440-6295

Fax: +82-2-440-6073

E-mail: leeshdr@khu.ac.kr

Keywords: Total mesorectal excision, Rectal neoplasms, Transanal approach

Supplementary video file: This article contains supplementary material (http://dx.doi.org/10.7602/ jmis.2016.19.2.79).

This is an Open Access article distributed under the terms of the Creative Commons Attribution Non-Commercial License (http:// creativecommons.org/licenses/by-nc/4.0/) which permits unrestricted non-commercial use, distribution, and reproduction in any medium, provided the original work is properly cited.

Copyright $\odot 2016$ The Journal of Minimally Invasive Surgery. All rights reserved.

\section{INTRODUCTION}

Laparoscopic resection for low rectal cancer showed a lower rate of positive circumferential margin (CRM) compared with open resection in COLOR II trial. ${ }^{1}$ It might be due to a better view of laparoscopy. However, total mesorectal excision (TME) for mid or low rectal cancer remains technically demanding and challenging under complicated conditions which are related with positive CRM or local recurrence even in the era of minimally invasive surgery. Transanal TME has been introduced as a novel option for mid or low rectal cancer. A few surgeons showed acceptable results of transanal TME for low rectal cancer compared with conventional abdominal approach. ${ }^{2-8}$ In addition, several studies comparing transanal TME with laparoscopic TME for mid and low rectal cancer are on-going. ${ }^{9}$ We demonstrate our procedure for transanal TME by using a short video clip.

\section{PROCEDURE}

A 70-year-old female patient was diagnosed with rectal adenocarcinoma. The location of the tumor was $2 \mathrm{~cm}$ from the anal verge and the clinical stage was T3N2 based on the initial $\mathrm{CT}$ and MRI. The patient underwent neoadjuvant chemora- 
diotherapy: short course radiotherapy $(5 \times 5 \mathrm{~Gy})$ with 5-fluorouracil (5-FU) and leucovorin (LV) chemotherapy. Additional 3 cycles of simplified infusional 5-FU/LV were given every 2 weeks to the patient during the resting period before the surgery (i.e. consolidation chemotherapy). After 8 weeks from the last radiotherapy, radical surgery was performed.

Surgery was composed of two parts: transanal total mesorectal excision and transabdominal dissection. After a pursestring suture of distal rectum just above the dentate line, vagina was identified to confirm the exact surgical plane. Transanal circumferential dissection including the mesorectum was performed from the dentate line until the peritoneal reflection, which can be determined when intraperitoneal organs are visible. Thereafter, transabdominal dissection was performed using laparoscopy. A medial to lateral approach starting at the aortic bifurcation was done and the inferior mesenteric artery was ligated at the pedicle. Lateral detachment and splenic flexure mobilization were completed. After full mobilization of the distal transverse colon and rectum, the specimen was retrieved through the anus and resected. Coloanal anastomosis was performed by hand-sewn method. A closed suction drain was inserted into the pelvis.

The patient recovered without any complications and was discharged on the postoperative day 8. Final pathology revealed as ypT3N1. She received adjuvant chemotherapy with FL and has been without any local or distant recurrence for the past 2 years.

\section{DISCUSSION}

Transanal TME was performed safely for the patient with low rectal cancer following neoadjuvant chemoradiotherapy. Recent studies showed reasonable data of transanal TME comparable to laparoscopic TME, in terms of postoperative complications and TME quality., ${ }^{4,10,11}$ Some authors suggested that transanal TME provided a better view in the deep pelvis because the surgeon's view and the plane of dissection were in the same axis. During conventional laparoscopic rectal transection, sometimes it is difficult to achieve a safe distal margin. However, transanal TME might improve quality of dissection and clear distal resection margin. It should be proven by further studies.

\section{REFERENCES}

1) van der Pas MH, Haglind E, Cuesta MA, et al. Laparoscopic versus open surgery for rectal cancer (COLOR II): short-term outcomes of a randomised, phase 3 trial. Lancet Oncol 2013;14:210-218.

2) Sylla P, Rattner DW, Delgado S, Lacy AM. NOTES transanal rectal cancer resection using transanal endoscopic microsurgery and laparoscopic assistance. Surg Endosc 2010;24:1205-1210.

3) Cahill RA, Hompes R. Transanal total mesorectal excision. Br J Surg 2015;102:1591-1593.

4) Fernandez-Hevia M, Delgado S, Castells A, et al. Transanal total mesorectal excision in rectal cancer: short-term outcomes in comparison with laparoscopic surgery. Ann Surg 2015;261:221227.

5) McLemore EC, Harnsberger CR, Broderick RC, et al. Transanal total mesorectal excision (taTME) for rectal cancer: a training pathway. Surg Endosc 2015(in press).

6) Burke JP, Martin-Perez B, Khan A, et al. Transanal total mesorectal excision for rectal cancer: early outcomes in 50 consecutive patients. Colorectal Dis 2016;18:570-577.

7) Funahashi K, Koike J, Teramoto T, et al. Transanal rectal dissection: a procedure to assist achievement of laparoscopic total mesorectal excision for bulky tumor in the narrow pelvis. Am J Surg 2009; 197:e46-50.

8) Marks J, Mizrahi B, Dalane S, Nweze I, Marks G. Laparoscopic transanal abdominal transanal resection with sphincter preservation for rectal cancer in the distal $3 \mathrm{~cm}$ of the rectum after neoadjuvant therapy. Surg Endosc 2010;24:2700-2707.

9) Rectal Cancer Surgery. COLOR III Trial: A randomized clinical trial comparing transanal and traditional laparoscopic TME for rectal cancer. Amsterdam: Rectal Cancer Surgery; 2016 [cited 2016 30 May]. Available from: http://rectalcancersurgery.eu/color3-trial/professionals/color-iii/.

10) Denost Q, Adam JP, Rullier A, Buscail E, Laurent C, Rullier E. Perineal transanal approach: a new standard for laparoscopic sphincter-saving resection in low rectal cancer, a randomized trial. Ann Surg 2014;260:993-999.

11) Velthuis S, Nieuwenhuis DH, Ruijter TE, Cuesta MA, Bonjer HJ, Sietses C. Transanal versus traditional laparoscopic total mesorectal excision for rectal carcinoma. Surg Endosc 2014;28:3494-3499. 